\title{
Comparison of Thiamin Diphosphate High-Performance Liquid Chromatography and Erythrocyte Transketolase Assays for Evaluating Thiamin Status in Malaria Patients without Beriberi
}

\author{
Andrew J. Taylor, ${ }^{1,2 \star}$ Dinesh Talwar, ${ }^{3}$ Sue J. Lee, ${ }^{2,4}$ Lorna Cox, ${ }^{5}$ Mayfong Mayxay, ${ }^{1,2,6}$ and Paul N. Newton ${ }^{1,2,4}$ \\ ${ }^{1}$ Lao Oxford-Mahosot Hospital-Wellcome Trust Research Unit (LOMWRU), Microbiology Laboratory, Mahosot Hospital, Vientiane, Lao PDR; \\ ${ }^{2}$ Centre for Tropical Medicine and Global Health, Churchill Hospital, University of Oxford, Oxford, United Kingdom; ${ }^{3}$ Scottish Trace Element \\ and Micronutrient Diagnostic and Research Laboratory, Glasgow Royal Infirmary, Glasgow, United Kingdom; ${ }^{4}$ Mahidol-Oxford Tropical \\ Medicine Research Unit, Faculty of Tropical Medicine, Mahidol University, Bangkok, Thailand; ${ }^{5}$ MRC Elsie Widdowson Laboratory, Cambridge, \\ United Kingdom; ' Institute of Research and Education Development, University of Health Sciences, Ministry of Health, Vientiane, Lao PDR
}

\begin{abstract}
Thiamin deficiency, or beriberi, is an increasingly re-recognized cause of morbidity and mortality in the developing world. Thiamin status has traditionally been measured through the erythrocyte activation assay (ETKA) or basal transketolase activity (ETK), which indirectly measure thiamin diphosphate (TDP). Thiamin diphosphate can also be measured directly by high-performance liquid chromatography (HPLC), which may allow a more precise estimation of thiamin status. We compared the direct measurement of TDP by HPLC with basal ETK activity and ETKA in 230 patients with Plasmodium falciparum malaria in rural southern Laos without overt clinical beriberi, as part of a trial of thiamin supplementation. Admission thiamin status measured by basal ETK activity and ETKA ( $\alpha$ ) were compared with thiamin status assessed by the measurement of TDP by HPLC. $55 \%$ of 230 included patients were male, and the median age was 10 (range $0.5-73$ ) years. Using $\alpha \geq 25 \%$ as the gold standard of thiamin deficiency, the sensitivity of TDP $<275 \mathrm{ng} / \mathrm{gHb}$ as a measure of thiamin deficiency was $68.5 \%(95 \% \mathrm{Cl}: 54.4-80.5 \%)$, with specificity of 60.8 (95\% Cl: $53.2-68.1 \%)$. There was a significant inverse correlation between the results of the two tests (Kendall's tau $=-0.212, P<0.001$ ). Basal ETK activity was also significantly positively correlated with TDP levels (Kendall's tau $=0.576, P<0.001$ ). Thiamin diphosphate measurement may have a role in measuring thiamin levels in clinical settings. Further studies evaluating TDP concentration in erythrocytes with basal ETK activity and ETKA $(\alpha)$ in beriberi patients would help establish comparative values of these assays.
\end{abstract}

\section{INTRODUCTION}

Until the 1950s, thiamin (vitamin B1, thiamine) deficiency was recognized as an important healthcare problem in the developing world with much public health research and debate. ${ }^{1}$ Incidence has since declined in wealthier areas, but there are increasing reports of infantile and adult thiamin deficiency in less wealthy areas of the world, especially in Asia. $^{2-6}$ The symptoms and signs of thiamin deficiency, or beriberi, include myocardial dysfunction, encephalopathy, and peripheral neuropathy, and may be fatal. However, beriberi is readily and inexpensively treatable with parenteral thiamin. Diagnosis of thiamin deficiency is almost always clinical, which is possible in patients with clear deficiency, but is more challenging in those with less marked symptoms or with concurrent infections. Clinically unapparent thiamin deficiency has been shown to be common among sick infants admitted to hospital in Vientiane ${ }^{2}$ and in Cambodian infants. ${ }^{4}$

Thiamin status has traditionally been assessed by indirect, functional measurement of thiamin diphosphate (TDP) in erythrocytes using the transketolase activation assay. ${ }^{7}$ This calculates the activation coefficient ( $\alpha$ ) by measuring the erythrocyte transketolase activity (ETK) before (basal) and after (activated) the addition of TDP in vitro. The transketolase activation assay, however, is limited by the expense and rare availability of the test, reference ranges which have not been standardized in children or by geographical regions, ${ }^{7-9}$ poor inter-assay replicability, sample storage instability due to rapid inactivation of the transketolase enzyme, and no external quality assurance systems. ${ }^{10}$ Furthermore, it has been demonstrated that the apoenzyme may not reactivate following

* Address correspondence to Andrew Taylor, Lao-Oxford-Mahosot Hospital-Wellcome Trust Research Unit (LOMWRU), Microbiology Laboratory, Mahosot Hospital, Vientiane, Laos. E-mail: andrewtaylor9@gmail.com chronic deficiency in vivo, ${ }^{10,11}$ binding of the apoenzyme and coenzyme may be altered by the presence of isoenzymes or other cofactors (e.g., magnesium), ${ }^{12}$ and synthesis may be reduced in those with diabetes and liver disease. ${ }^{13,14}$ Recently, it has been suggested that basal erythrocyte ETK activity is a more accurate biomarker of infantile beriberi than the activation coefficient, ${ }^{3}$ although there are no recognized reference ranges established for basal ETK activity.

An alternative method for the evaluation of thiamin status is the direct measurement of erythrocyte TDP by high-performance liquid chromatography (HPLC). ${ }^{15}$ Thiamin diphosphate concentrations in erythrocytes are good indicators of body stores, ${ }^{16}$ and the method has been shown to be accurate and easier to be standardized than ETKA. ${ }^{15,17}$ However, the TDP method shares the disadvantages of ETKA ( $\alpha$ ) in terms of having reference ranges that are not standardized in children or by geographical region. Current reference ranges for TDP are descriptive of a "normal population" and not based on physiology. Furthermore, the relationship between ETKA and TDP $(\alpha)$ is not clear.

Data for this study come from a trial of malaria supplementation in southern Laos. ${ }^{18}$ Malaria is a common cause of morbidity in southern Laos, and one third of patients present with biochemical thiamin deficiency. ${ }^{18}$ It was postulated thiamin supplementation may improve neurological side effects and adverse outcomes. ${ }^{5,18}$ The trial demonstrated that thiamin levels increased following supplementation, but there was no reduction in adverse events. Here, we compare the assessment of thiamin status by direct measurement of TDP using HPLC against erythrocyte transketolase assays in these malaria patients. ${ }^{18}$

\section{METHODS}

Patient cohort. We compared admission TDP concentration with ETKA ( $\alpha$ ) and basal ETK activity in erythrocytes in 230 
patients infected with Plasmodium falciparum, as demonstrated by positive admission Giemsa-stained peripheral blood slides, in southern Laos; see ref. 18 for details. Samples were collected during the rainy season, when malaria transmission is high, from June to November 2008 to 2010. None of the patients had documented, clinically apparent, thiamin deficiency and none were known to have received thiamin supplementation. No patients were fasted. Written informed consent was obtained from all participants. Ethical clearance for the study was granted by the Lao National Ethics Committee for Health Research and the Oxford University Tropical Medicine Research Ethics Committee.

Blood sample preparation. Immediately after venous blood collection, $1 \mathrm{~mL}$ lithium heparin anticoagulated blood was centrifuged and washed in phosphate-buffered saline three times, with removal of the buffy coat initially and again after each wash. Washed erythrocyte samples were stored at $-30^{\circ} \mathrm{C}$ for maximum of 3 months and then at $-80^{\circ} \mathrm{C}$ until shipment to the United Kingdom on dry ice.

Erythrocyte transketolase activity assays. Erythrocyte transketolase activity assays were performed at the Medical Research Council Elsie Widdowson Laboratory in Cambridge, United Kingdom, using an adaptation of the method of Vuilleumier et al. ${ }^{19}$ as described in Khounnorath et al. ${ }^{2}$ and Soukaloun et al. $^{3}$ These are functional assays for the thiamindependent erythrocyte transketolase enzyme in washed red cells. The assays were performed in microplate format in a temperature-controlled instrument which provided a consistent temperature over the whole plate (Multiskan, Thermo Fisher Scientific, Waltham, MA); 20 samples and three single-donor washed erythrocyte quality controls (QC), prepared in-house, were assayed on each plate. All QC materials were obtained from persons with adequate thiamin status; no "deficient" samples were available. Hemoglobin $(\mathrm{Hb})$ was measured in the same lysate using Zap-o-globin (Beckman-Coulter, Brea, CA).

The activation coefficient in vitro may be expressed either as the ratio of ETK activity after activation with excess TDP to basal ETK activity or as $\alpha$. $\alpha$ is the ratio of in vitro ETK activity after TDP has been added minus the basal ETK activity before TDP has been added, to the basal ETK activity, expressed as a percentage. Expressing ETKA as a greatly magnifies the discrimination. Higher ETKA ratio or a represents a greater degree of thiamin deficiency. The threshold for biochemical thiamin insufficiency is regarded as a ratio $>1.25$ or its equivalent, $\alpha=25$. In this study, thiamin deficiency was defined as $\alpha \geq 25 .{ }^{18}$ Basal ETK activity of $0.59 \mathrm{per} \mathrm{g} / \mathrm{Hb}$ was used as a cutoff value to signify biochemical deficiency. ${ }^{3}$ The between-batch imprecision of the assay was $14-20 \%$ for ETKA expressed as a (3-5\% when expressed as ratio), and $7-10 \%$ for ETK/gHb.

Thiamin diphosphate measurement. Thiamin diphosphate measurement was performed at the Scottish Trace Element and Micronutrient Diagnostic and Research Laboratory. Erythrocytes were prepared as described for the transketolase assay, and TDP was measured by HPLC using post-column derivation with alkaline potassium ferricyanide as described in the study by Talwar et al. ${ }^{15}$ The same blood was drawn, but different aliquots were used for the two assays. Internal QC samples were used; QC1 was hemolysate, and QC2 was prepared by addition of TDP solution to the hemolysate. Six QCs were run with each batch of 30 samples: two at the start, two in the middle, and two at the end of the run. A lower limit of the adult reference range of $275 \mathrm{ng} / \mathrm{gHb}$
TDP was used based on the lower 2.5 percentile of concentrations found in Talwar's "normal" population. ${ }^{15}$ This value was not based on physiological considerations or requirements for thiamin. The between-batch CV of the assay was $<7 \%$.

Statistical analysis. Graphs were plotted comparing erythrocyte TDP concentrations with ETKA $(\alpha)$ and basal ETK activity. Kendall's rank correlation coefficient $(\mathrm{T})$ was calculated to assess the association between the continuous values of TDP, ETKA ( $\alpha$ ), and basal ETK activity. Sensitivity and specificity of TDP $\geq 275 \mathrm{ng} / \mathrm{gHb}$ were calculated using $\alpha \geq 25 \%$ as the gold standard, as this has been the established method for measuring thiamin status. "Ideal" TDP cutoffs were estimated using $\alpha \geq 25 \%$ and basal ETK activity $<0.59$ as the reference variables to indicate those with "true" thiamin deficiency. Receiver operating characteristic (ROC) curves were plotted, and the "ideal" cutoff was defined as the point closest to the top left corner of the graphs.

\section{RESULTS}

Samples from 230 patients were included, of whom 55\% were male. The median age was 10 (range 0.5-73) years, with 78 patients aged $>16$ and 152 patients aged $<16$ years.

Of 230 patients, 106 had erythrocyte TDP levels $<275 \mathrm{ng} /$ $\mathrm{gHb}$. Of these, 34.9\% (37/106) also had $\alpha$ levels $\geq 25 \%$, whereas $65.1 \%(69 / 106)$ had a levels $<25 \%$. One hundred twenty-four patients had erythrocyte TDP levels $\geq 275 \mathrm{ng} /$ $\mathrm{gHb}$, of which $86.3 \%(107 / 124)$ also had $\alpha$ levels $<25 \%$ and $13.7 \%(17 / 124)$ had $\alpha$ levels $\geq 25 \%$. Fifty-four patients had $\alpha$ levels $\geq 25 \%$, of which $68.5 \%$ (37/54) also had erythrocyte TDP levels $<275 \mathrm{ng} / \mathrm{gHb}$ (Table 1).

Using $\alpha \geq 25 \%$ as the gold standard of biochemical thiamin deficiency, the sensitivity of erythrocyte TDP $<275 \mathrm{ng} / \mathrm{gHb}$ as a measure of thiamin deficiency was $68.5 \%(95 \% \mathrm{Cl}$ : $54.4-80.5 \%$ ) with specificity of 60.8 (95\% Cl: 53.2-68.1\%). There was a significant inverse correlation between the tests (Kendall's tau $=-0.212, P<0.001$ ). Inverse correlation was also observed between the two assays when adults (Supplemental Figure 2) (78 patients, Kendall's tau $=-0.227, P=0.003$ ) and children (Supplemental Figure 1) (152 patients, Kendall's tau $=-0.176, P=0.001$ ) were analyzed separately.

Basal ETK activity was significantly positively correlated with erythrocyte TDP levels (Kendall's tau $=0.576, P<0.001$, Figure 2, Table 2, Supplemental Figure 3). Of 106 patients with TDP levels < $275 \mathrm{ng} / \mathrm{gHb}, 98.1 \%(104 / 106)$ had basal ETK activity $<0.59 \mu$ moles $/$ minute/gHb. Of 179 patients with a basal ETK activity $<0.59 \mu$ moles/minute/gHb, 58.1\% (104/179) had TDP levels $<275 \mathrm{ng} / \mathrm{gHb}$. A correlation was also demonstrated when adults (78 patients, Kendall's tau $=0.597, P<0.001$ ) and children (152 patients, Kendall's tau $=0.546, P<0.001$ ) were analyzed separately. Basal ETK was significantly lower in adults than in children aged $<16$ years $(0.44$ versus $0.52, P=0.003)$, as was TDP (265 versus $314, P=0.003$ ).

TABLE 1

Frequency of thiamin deficiency using admission $\alpha \geq 25 \%$ and erythrocyte TDP $<275 \mathrm{ng} / \mathrm{gHb}$ in 230 malaria patients

\begin{tabular}{lccr}
\hline & TDP $<275$ & TDP $\geq 275$ & Total \\
\hline$\alpha \geq 25 \%$ & 37 & 17 & 54 \\
$\alpha<25 \%$ & 69 & 107 & 176 \\
Total & 106 & 124 & 230 \\
\hline
\end{tabular}




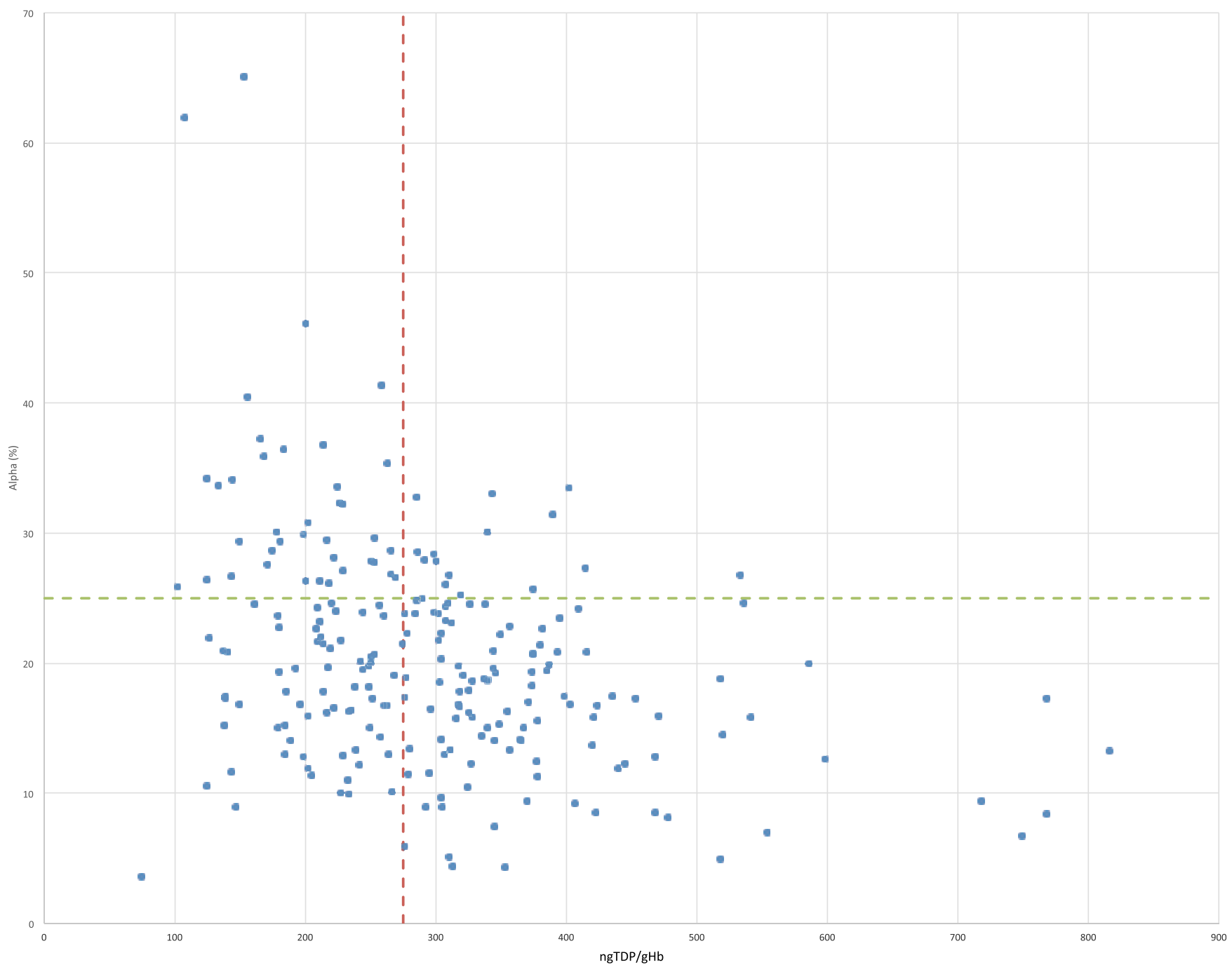

FIGURE 1. Relationship between admission alpha and thiamin diphosphate for 230 malaria patients. This figure appears in color at www.ajtmh.org.

Nonparametric ROC curves estimated that the optimum erythrocyte TDP value for $\alpha \geq 25 \%$ prediction was $<269 \mathrm{ng} /$ $\mathrm{gHb}$, which gave a sensitivity and specificity of $68.5 \%$ and $61.4 \%$, respectively; this resulted in $63.0 \%$ of patients being correctly classified (area under the ROC [AUROC] curve = 0.6820 ). For basal ETK activity $<0.59 \mu$ moles/minute/gHb, a TDP cutoff of $<321 \mathrm{ng} / \mathrm{gHb}$ gave a sensitivity of and specificity of $78.8 \%$ and $80.4 \%$, respectively, and resulted in $79.1 \%$ of patients being correctly classified (AUROC curve $=0.8881$ ).

\section{DISCUSSION}

This study compared erythrocyte TDP concentration with ETKA ( $\alpha$ ) to evaluate biochemical thiamin status in malaria patients without clinical beriberi. Results showed that TDP concentration in erythrocytes was inversely correlated with ETKA $(\alpha)$ in both adults and children. When comparing the tests' ability to define biochemical thiamin deficiency (comparing erythrocyte TDP $<275 \mathrm{ng} / \mathrm{gHb}$ with $\alpha$ levels $>25 \%$ ), TDP had a sensitivity of $68.5 \%$ (95\% Cl: $54.4-80.5 \%)$ and a specificity of $60.8 \%$ (95\% Cl: 53.2-68.1\%). The correlation between basal ETK activity and erythrocyte TDP concentration was stronger than the correlation between $\alpha$ and erythrocyte TDP $(0.576$ versus -0.212 , respectively, $P<0.001)$.

Both ETKA and TDP assays have limitations. Thiamin diphosphate is not a metabolic functional endpoint and does not determine whether the thiamin concentration present is sufficient to saturate the enzyme. Furthermore, the lower reference range of erythrocyte TDP ( $275 \mathrm{ng} / \mathrm{gHb})$ used in this study was not based on physiological considerations or human requirements for thiamin. ETKA may therefore have a theoretical advantage in measuring thiamin status, as it is a functional measure.

Limitations of this study include the fact that no patients in the cohort manifested clinical signs of thiamin deficiency, so it is not possible to correlate results with clinical thiamin deficiency. It is possible that some patients had subclinical deficiency. Determining a clinical endpoint for thiamin deficiency is challenging, as beriberi seems to occur not only when thiamin deficiency exists but also when additional exacerbating 
Day 0 Basal activity vs TDP

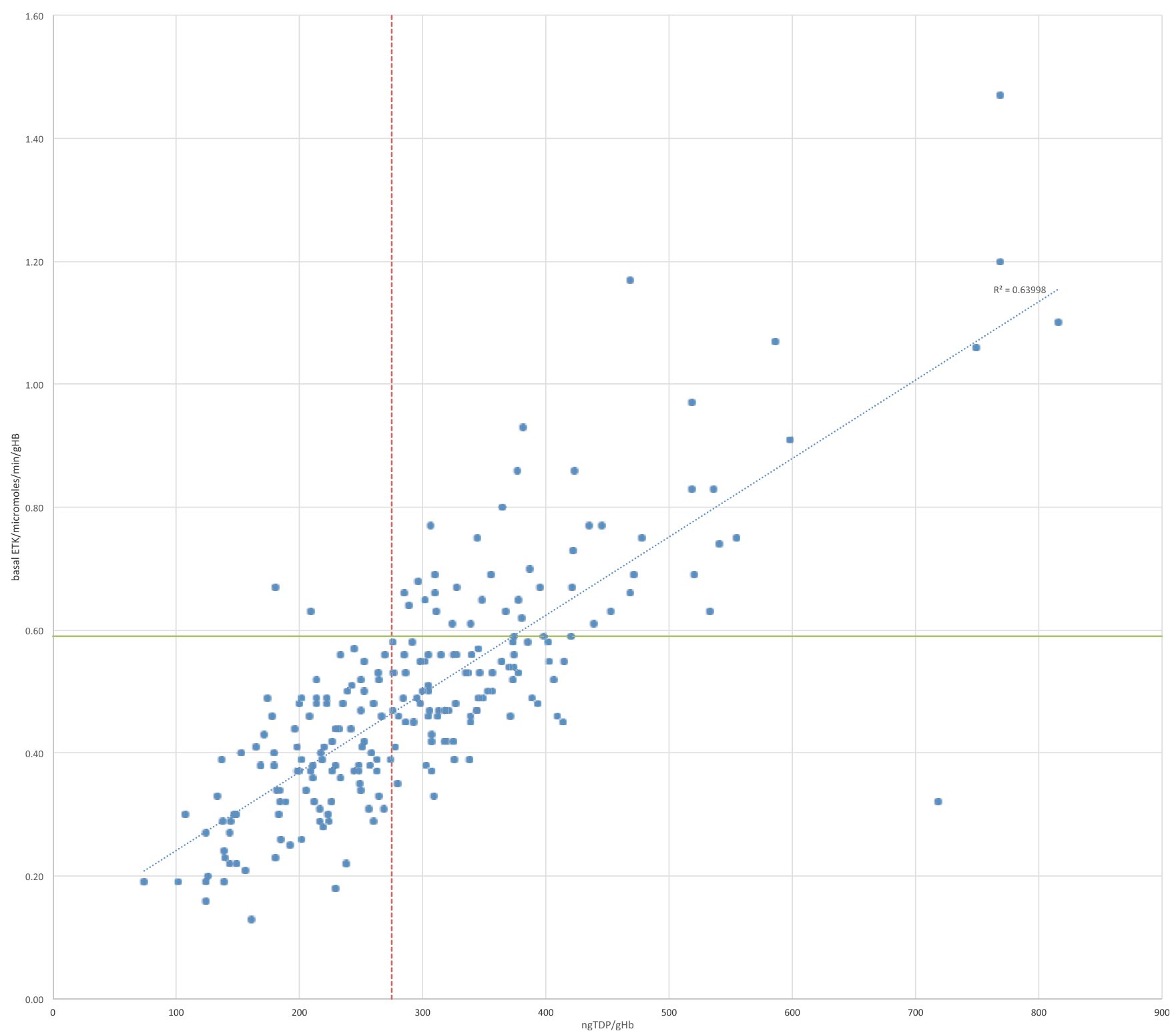

FIGURE 2. Relationship between admission basal ETK and thiamin diphosphate for 230 malaria patients. This figure appears in color at www.ajtmh.org.

factors such as infections are present. The distinction between those who do and do not show clinical symptoms of beriberi at different biochemical assay levels may also be confounded by other factors. Importantly, these data cannot

TABLE 2

Frequency of admission basal erythrocyte ETK activity $<0.59 \mu$ moles/ minute/g $\mathrm{Hb}$ and TDP concentration $<275 \mathrm{ng} / \mathrm{gHb}$ for 230 malaria patients

\begin{tabular}{lccr}
\hline & TDP $<275$ & TDP $\geq 275$ & \\
\hline Basal ETK activity $<0.59$ & 104 & 75 & 179 \\
Basal ETK activity $\geq 0.59$ & 2 & 49 & 51 \\
& 106 & 124 & 230 \\
\hline
\end{tabular}

TDP $=$ thiamin diphosphate be used to inform biochemical diagnosis of infantile beriberi as children aged $<6$ months were not included.

Further work is required to understand the relationship between erythrocyte TDP levels and ETKA with clinical features of beriberi and patient outcomes. Measurement of TDP in erythrocytes showed a clear linear correlation with basal ETK activity (Figure 2), suggesting it may have a role in measuring thiamin status. Further studies comparing TDP concentration, basal ETK activity, and ETKA ( $\alpha$ ) in patients with and without beriberi, before and after thiamin supplementation, would help define reference levels for biochemical thiamin deficiency for different patient ages and establish the comparative values of these assays. 
Received May 14, 2020. Accepted for publication August 4, 2020.

Published online September 28, 2020.

Note: Supplemental figures appear at www.ajtmh.org.

Acknowledgments: We are very grateful to the participants of this study, and to the doctors, medical assistants, and nurses in Xepon Interdistrict Hospital. We are very grateful to Bounthaphany Bounxouei, past director of Mahosot Hospital; to Bounnack Saysanasongkham, director of Department of Health Care, Ministry of Health; to H. E. Bounkong Syhavong, Minister of Health, Lao PDR; to the director and staff of the Centre of Malariology, Parasitology, and Entomology, Vientiane; and to Director and the staff of the Microbiology Laboratory of Mahosot Hospital. We would like to thank the staff at the Scottish Micronutrient Reference Laboratory, Glasgow, Scotland, who performed the TDP assay, and staff at the UKRI-Medical Research Council (MRC) Elsie Widdowson Laboratory, Cambridge, United Kingdom, who performed the transketolase assay. We would like to thank Denis O'Reilly for his advice and input.

Financial support: Lao Oxford-Mahosot Hospital-Wellcome Trust Research Unit (LOMWRU) is supported by a Wellcome Trust grant.

Authors' addresses: Andrew J. Taylor, Centre for Tropical Medicine and Global Health, Churchill Hospital, University of Oxford, Oxford, United Kingdom, E-mail: andrewtaylor9@gmail.com. Dinesh Talwar, Department of Clinical Biochemistry, Glasgow Royal Infirmary, Glasgow, United Kingdom, E-mail: dinesh.talwar@ggc.scot.nhs.uk. Sue J. Lee, Mahidol-Oxford Tropical Medicine Research Unit, Mahidol University, Bangkok, Thailand, and Centre for Tropical Medicine and Global Health, Churchill Hospital, University of Oxford, Oxford, United Kingdom, E-mail: sue@tropmedres.ac. Lorna Cox, MRC Epidemiology Unit, University of Cambridge, Cambridge, United Kingdom, E-mail: lorna.cox@mrc-epid.cam.ac.uk. Mayfong Mayxay and Paul N. Newton, Lao Oxford-Mahosot Hospital-Wellcome Trust Research Unit (LOMWRU), Microbiology Laboratory, Mahosot Hospital, Vientiane, Laos, and Centre for Tropical Medicine and Global Health, Churchill Hospital, University of Oxford, Oxford, United Kingdom, E-mails: mayfong@tropmedres.ac and paul.newton@tropmedres.ac.

This is an open-access article distributed under the terms of the Creative Commons Attribution (CC-BY) License, which permits unrestricted use, distribution, and reproduction in any medium, provided the original author and source are credited.

\section{REFERENCES}

1. Carpenter K, 2000. Beriberi, White Rice, and Vitamin B: A Disease, a Cause, and a Cure. Berkeley, CA: University of California Press.

2. Khounnorath $S$ et al., 2011. Clinically unapparent infantile thiamin deficiency in Vientiane, Laos. PLoS Negl Trop Dis 5: e969.
3. Soukaloun D et al., 2011. Erythrocyte transketolase activity, markers of cardiac dysfunction and the diagnosis of infantile beriberi. PLoS Negl Trop Dis 5: e971.

4. Coats $D$ et al., 2012. Thiamine deficiency in Cambodian infants with and without beriberi. J Pediatr 161: 843-847.

5. Krishna S, Taylor AM, Supanaranond W, Pukrittayakamee S, Ter Kuile F, Tawfiq KM, Holloway PAH, White NJ, 1999. Thiamine deficiency and malaria in adults from southeast Asia. Lancet 353: 546-549.

6. Nilles EJ et al., 2018. Re-emergence of thiamine deficiency disease in the Pacific Islands (2014-15): a case-control study. PLoS One 13: 1-14.

7. Dreyfus $P, 1962$. Clinical application of blood transketolase determinations. N Engl J Med 267: 596-598.

8. Brin M, 1962. Erythrocyte transketolase in early thiamine deficiency. Ann N Y Acad Sci 98: 528-541.

9. Sauberlich H, 1967. Biochemical alterations in thiamine deficiencytheir interpretation. Am J Clin Nutr 17: 528-542.

10. Puxty J, Haskew A, Ratcliffe J, McMurray J, 1985. Changes in erythrocyte transketolase activity and the thiamin pyrophosphate effect during storage of blood. Ann Clin Biochem 22: 423-427.

11. Wood B, Breem K, Pennington D, Wood B, Breen K, Pennington D, 1977. Thiamine status in alcoholism. Aust N Z J Med 7: 475-484.

12. Kaczmarek M, Nixon $P, 1983$. Variants of transketolase from human erythrocytes. Clin Chim Acta 130: 249-256.

13. Friedrich W, 1988. Vitamins. Berlin, Germany: De Gruyter, 341-394.

14. Fennelly J, Frank O, Baker H, Leevy CM, 1967. Red blood celltransketolase activity in malnourished alcoholics with cirrhosis. Am J Clin Nutr 20: 946-949.

15. Talwar D, Davidson H, Cooney J, JO’Reilly DS, 2000. Vitamin B1 status assessed by direct measurement of thiamin pyrophosphate in erythrocytes or whole blood by HPLC: comparison with erythrocyte transketolase activation assay. Clin Chem 46: 704-710.

16. Brin M, 1964. Erythrocyte as biopsy tissue for functional evaluation of thiamin adequacy. JAMA 187: 762-766.

17. Baines M, Davies G, 1988. The evaluation of erythrocyte thiamin diphosphate as an indicator of thiamin status in man, and its comparison with erythrocyte transketolase activity measurements. Ann Clin Biochem 25: 698-705.

18. Mayxay M et al., 2014. Thiamin supplementation does not reduce the frequency of adverse events after anti-malarial therapy among patients with falciparum malaria in southern Laos. Malar J 13: 1-13.

19. Vuilleumier J, Keller H, Neck E, 1990. Clincal chemical methods for the routine assessment of the vitamin status of human populations. Int J Vitam Nutr Res 60: 126-135. 